\title{
Mother-to-infant transmission of HIV-1: the placenta fights back
}

\author{
Stephen A. Spector
}

Department of Pediatrics, Division of Infectious Diseases, Center for Molecular Genetics and Center for AIDS Research, University of California-San Diego, Stein Clinical Research Building, 9500 Gilman Drive, La Jolla, California 92093-0672, USA. Phone: (858) 534-7170; Fax: (858) 534-7411; E-mail: saspector@ucsd.edu.

Much progress has been made in preventing the transmission of HIV-1 from an infected mother to her infant. In the landmark Pediatric AIDS Clinical Trials Group (PACTG) 076 study, mother-to-infant transmission was decreased from approximately $25 \%$ to $8 \%$ (1). In subsequent studies, various antiretrovirals used singly or in combination when administered to a pregnant woman and her infant have further impacted transmission (2-4). Remarkably, even a single dose of the non-nucleoside reverse transcriptase inhibitor nevirapine, when administered during labor and to an infant shortly after birth, can reduce vertical transmission by 50\% (5). With the availability of antiprotease inhibitors used in combination with other antiretrovirals, transmission can be reduced to less than $2 \%$. In spite of these advances, it is estimated that daily 1,600 infants are still being infected with HIV-1 perinatally worldwide (6). Thus, understanding the risk factors associated with mother-to-infant transmission remains critically important for the development of improved strategies for the prevention of vertical transmission.

Despite the remarkable improvements in the use of antiretrovirals for the prevention of mother-to-infant HIV-1 transmission, little is known of factors that impact vertical transmission, and still less is known on the role of the placenta. How the placenta might prevent the fetus from infection has been questioned not only for HIV-1 but also for many other viruses that can cause congenital infection, including rubella and cytomegalovirus (CMV), where maternal infection does not invariably correlate with infection of the fetus. In this issue of the JCI, Patterson and his colleagues present data to suggest that leukemia inhibitory factor (LIF) inhibits HIV-1 replication prior to reverse transcription, possibly through signal transduction through the JAK/STAT pathway, and is upregulated in the placentas of nontransmitting women (7). LIF, a member of the IL- 6 cytokine family, maintains the pluripotency of embryonic stem cells in vitro, and LIF knockout mice are unable to implant blastocysts, resulting in infertility (8). The receptor for LIF (LIFR) is thought to play a role in trophoblast growth and differentiation in the human placenta. In human placenta, cells of the trophoblast lineage express LIFR and the major site of LIF mRNA expression in leukocytes is the maternal decidua (9). Patterson et al. (7) find that the level of LIF mRNA and the presence of LIF protein expressing cells are significantly greater in nontransmitting mothers compared with transmitters. Thus, the suggestion is that the placentas of some women express lower quantities of LIF and increase the risk of mother-toinfant transmission.

Factors that have previously been shown to increase the risk of mother-toinfant transmission include advanced HIV-1-related illness/AIDS, low CD4 ${ }^{+}$ lymphocyte counts, high HIV-1 viral load, the presence of sexually transmitted diseases, viral phenotype, prolonged rupture of amniotic membranes, and absence of maternal autologous neutralizing antibody $(10,11)$. However, even at the highest viral loads, no more than half of exposed infants become infected with HIV-1. Moreover, considerable data support that greater than two-thirds of non-breast-milk-related transmission occurs during labor in the intrapartum period. Thus, the placenta as well as other factors must be important in preventing in utero transmission.

A number of genetic factors have been found to impact mother-toinfant transmission, including polymorphisms in the chemokine receptor CCR5 gene. To my knowledge, no HIV-1 infected infant has been identified as homozygous for the CCR5 $\Delta 32$ polymorphism (12, 13). However, whereas there appears to be no protection afforded to the $\Delta 32$ heterozy- gote, a polymorphism in the CCR5 promoter at position 59356 appears to alter the risk of perinatal transmission (14). Also, polymorphisms in genes encoding for chemokines such as the SDF1 $3^{\prime} \mathrm{A} / \mathrm{wt}$ genotype have been associated with increased transmission (15). Similarly, the more discordant the HLA type of a mother and her infant, the less likely HIV-1 is to be transmitted (16). Viral factors have also been identified in HIV-1 vertical transmission (17). In particular, CCR5-using virus (non-syncytiuminducing, macrophage-tropic) is most commonly transmitted to infants even when the predominant virus found in the mother uses the CXCR4 receptor. Additionally, most data suggest that an apparent minor population of maternal virus is transmitted from a mother to her infant when transmission does occur (18).

Although the findings of Patterson et al. (7) are provocative, how LIF affects transmission of HIV-1 is unclear. LIF must be administered to susceptible cells prior to infection. Thus, presumably, once cells are infected with HIV-1, LIF provides some protection only to the uninfected cells and not to the infected cells. Additionally, although it is true that LIF-producing cells are decreased in nontransmitting placentas, normal uninfected placentas produce the greatest amounts of LIF (although not to a statistically significant degree). Thus, whether HIV-1 infection of the placenta decreases production of LIF, making transplacental transmission of virus more likely, or whether the constitutive expression of LIF is lower in some placentas than others, is unclear. De et al. have made a similar observation for human chorionic gonadotropin (hCG) (19). In vitro, hCG can suppress HIV-1 reverse transcription in infected lymphocytes and monocytes and can block viral transmission resulting from cell-tocell contact between HIV-1-infected lymphocytes and placental trophoblasts. 
Selected Cytokines/Hormones Potentially Impacting on HIV-1 Transplacental Transmission

- IL-4

- Human chorionic gonadotropin

- Leukemia inhibitory factor

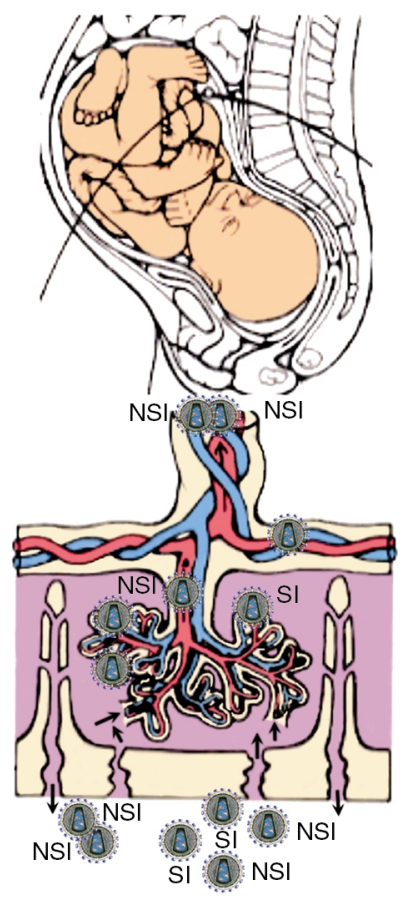

Selected Maternal/Infant Factors Increasing Risk of HIV-1 Transplacental Transmission

- High maternal HIV-1 load

- Low maternal CD4+ count

- NSI, macrophage-tropic, CXCR5-using virus

- CCR5 59356 T/T polymorphism (infant)

- CCR5 $\Delta 32 / \Delta 32$ (infant - prevents transmission)

- SDF1 $3^{\prime}$ A/wt polymorphism (mother)

- IL-4 promoter 589T polymorphism

- HLA concordance

\section{Figure 1}

Mother-to-infant transmission of HIV-1. Increasing evidence indicates that transplacental transmission of HIV-1 is the result of a complex interaction between genetic factors of the virus, mother, and infant. Maternal high virus load and low CD4+ lymphocyte count are most often associated with transmission with CCR5-using virus (non-syncytium-inducing [NSI], macrophage-tropic), most often transmitted even when CXCR4using virus (syncytium-inducing [SI], T cell-tropic) is present in the mother. Host genetic factors of mother and infant are also important determinants of transmission. Polymorphisms in genes encoding for the chemokine receptor CCR5 in the infant can decrease the risk of transmission. Also, polymorphisms in genes encoding for cytokines and hormones such as LIF, hCG, and IL-4 likely contribute to the placental barrier to viral transmission. Figure adapted in part from ref. 21.

A model of HIV-1 mother-to-infant transmission appears to be emerging that includes both innate and acquired protection (Figure 1). As Patterson et al. note, the establishment and maintenance of pregnancy are thought to be dependent in part on the balance of type 1 (Th1) and type 2 (Th2) cytokine responses (7). Progesterone promotes the development of T-helper cells that produce Th2 cytokines including IL-4 and IL-10. IL-4 is of particular interest because in vitro it promotes the growth of syncytium-inducing (SI) virus by increasing CXCR4 expression on $\mathrm{CD} 4^{+}$lymphocytes and decreasing CCR5 expression, and by stimulating replication of HIV-1 isolates via transcriptional activation (19). Moreover, recent data suggest that homozygosity of a polymorphism in the IL-4 promoter region, IL-4 589T, is correlated with increased rates of SI variant acquisition in HIV-1-infected individuals (20). Thus, polymorphisms in the IL-4 gene resulting in increased levels of IL-4 place pressure on virus to become SI, CXCR4-using strains that are least likely to be transmitted from mother to infant. At the same time, other genetic polymorphisms, including those impacting CCR5 expression, hCG, and LIF, may impact the likelihood of vertical transmission. Thus, it would seem that HIV-1 transmission from an infected mother to her infant is the result of a complex interplay between virus and host in which genotypes of virus, mother, and infant impact the risk of transmission. Natural defenses afforded through cytokines such as LIF constitutively expressed in the placenta likely provide considerable protection from transplacental transmission in most cases. In this regard, although it is not surprising that LIF failed to inhibit infection by Epstein-Barr virus, which rarely causes congenital infection and requires $\mathrm{CD} 21$ as its receptor, it is somewhat surprising that LIF had no inhibitory effect on infection by CMV, since one might anticipate that similar innate mechanisms are responsible for preventing transplacental transmission of congenitally affecting viruses.
In this regard, the use of monocytes that are naturally infected by CMV as the system for evaluation of LIF inhibition instead of infection of fibroblasts might demonstrate inhibitory activity of LIF against CMV.

The identification by Patterson and colleagues (7) of another innate substance that inhibits HIV-1 replication opens an additional line of attack for inhibiting mother-to-infant transmission of HIV-1. The application of these novel approaches may further increase the ability to efficiently and safely interrupt transmission of HIV-1 from an infected mother to her infant.

\section{Acknowledgments}

S.A. Spector's research is supported by grants from the NIH, including AI39004, AI-27563, and AI-36214.

1. Connor, E.M., et al. 1994. Reduction of maternal-infant transmission of human immunodeficiency virus type 1 with zidovudine treatment. N. Engl.J. Med. 331:1173-1180.

2. Wade, N.A., et al. 1998. Abbreviated regimens of zidovudine prophylaxis and perinatal transmission of the human immunodeficiency virus. $N$. Engl. J. Med. 339:1409-1414. 
3. Stiehm, E.R., et al. 1999. Efficacy of zidovudine and human immunodeficiency virus (HIV) hyperimmune immunoglobulin for reducing perinatal HIV transmission from HIV-infected women with advanced disease: results of Pediatric AIDS Clinical Trials Group protocol 185. J. Infect. Dis. 179:567-575.

4. Lallemant, M., et al. 2000. A trial of shortened zidovudine regimens to prevent mother-to-child transmission of human immunodeficiency virus type 1. Perinatal HIV Prevention Trial (Thailand) Investigators. N. Engl. J. Med. 343:982-991.

5. Guay, L.A., et al. 1999. Intrapartum and neonatal single-dose nevirapine compared with zidovudine for prevention of mother-to-child transmission of HIV-1 in Kampala, Uganda: HIVNET 012 randomised trial. Lancet. 354:795-802.

6. World Health Organization. 1998. Global summary of the HIV/AIDS epidemic update: December 1998. World Health Organization/UNAIDS AIDS. $1-18$.

7. Patterson, B.K., et al. 2001. Leukemia inhibitory factor inhibits HIV-1 replication and is upregulated in placentae from nontransmitting women. J. Clin. Invest. 107:287-294.

8. Stewart, C.L., et al. 1992. Blastocyst implantation depends on maternal expression of leukaemia inhibitory factor. Nature. 359:76-79.
9. Sharkey, A.M., et al. 1999. Localization of leukemia inhibitory factor and its receptor in human placenta throughout pregnancy. Biol Reprod. 60:355-364.

10. Sperling, R.S., et al. 1996. Maternal viral load, zidovudine treatment, and the risk of transmission of human immunodeficiency virus type 1 from mother to infant. Pediatric AIDS Clinical Trials Group Protocol 076 Study Group. N. Engl. J. Med. 335:1621-1629.

11. Mofenson, L.M., et al. 1999. Risk factors for perinatal transmission of human immunodeficiency virus type 1 in women treated with zidovudine. Pediatric AIDS Clinical Trials Group Study 185 Team. N. Engl. J. Med. 341:385-393.

12. Edelstein, R.E., et al. 1997. Risk of mother-toinfant transmission of HIV-1 is not reduced in CCR5-delta-32ccr5 heterozygotes. J. Acquir Immune Defic. Synd Hum. Retrovirol. 16:243-246.

13. Barroga, C.F., et al. 2000. The CCR5 $\Delta$ allele slows disease progression of human immunodeficiency virus (HIV) type-1 infected children receiving antiretroviral treatment. J. Infect. Dis. 182:413-419.

14. Kostrikis, L., et al. 1999. A polymorphism in the regulatory region of the $\mathrm{CC}$-chemokine receptor 5 gene influences perinatal transmission of human immunodeficiency virus type 1 to AfricanAmerican infants. J. Virol. 73:10264-10271.
15. John, G.C., et al. 2000. Maternal SDF1 3'A polymorphism is associated with increased perinatal human immunodeficiency virus type 1 transmission. J. Virol. 74:5736-5739.

16. MacDonald, K.S., et al. 1998. Mother-child class I HLA concordance increases perinatal human immunodeficiency virus type 1 transmission. $J$. Infect. Dis. 177:551-556.

17. Spencer, L.T., Ogino, M.T., Dankner, W.M., and Spector, S.A. 1994. Clinical significance of human immunodeficiency virus type 1 phenotypes in infected children. J. Infect. Dis. 169:491-495.

18. Wolinsky, S.M., et al. 1992. Selective transmission of human immunodeficiency virus type-1 variants from mothers to infants. Science. 255:1134-1137.

19. De, S.K., et al. 1997. Human chorionic gonadotropin hormone prevents wasting syndrome and death in HIV-1 transgenic mice. J. Clin. Invest. 99:1484-1491.

20. Nakayama, E.E., et al. 2000. Polymorphism in the interleukin-4 promoter affects acquisition of human immunodeficiency virus type 1 syncytium-inducing phenotype. J. Virol. 74:5452-5459.

21. Blanche, S. 1994. HIV in infants and children transmission and progression. In HIV: advances in research and therapy. Cliggot Communications. Greenwich, Connecticut, USA. 4:9-13. 